\title{
Effect of sequentially fed high protein, hydrolysed protein, and high fibre diets on the faecal microbiota of healthy dogs: a cross-over study
}

\author{
Lina Maria Martinez-Lopez \\ University of Melbourne

\section{Amy Pepper} \\ University of Melbourne \\ Rachel Pilla \\ Texas A\&M University College Station

\section{Andrew Woodward} \\ University of Melbourne \\ Jan Suchodolski \\ Texas A\&M University College Station \\ Caroline Mansfield ( $\nabla$ cmans@unimelb.edu.au ) \\ University of Melbourne https://orcid.org/0000-0002-5527-7096
}

\section{Research Article}

Keywords: Dog microbiota, diet, high-insoluble fibre, hypoallergenic, high-protein, hydrolysed

Posted Date: January 19th, 2021

DOI: https://doi.org/10.21203/rs.3.rs-61792/v2

License: (c) (i) This work is licensed under a Creative Commons Attribution 4.0 International License. Read Full License 


\section{Abstract}

\section{Background}

Dietary content and environmental factors can shape the gut microbiota, and consequently, the way the gut microbiota metabolises fats, carbohydrates and proteins, affecting overall health of the host. We evaluated the impact of 3 diets (all meat, high insoluble fibre dry extruded diet and hydrolysed protein dry extruded diet) on the gut microbiota of healthy dogs in a cross-over sequential study.

\section{Results}

We showed that diet can have a large effect on the gut microbiome in dogs, regardless of the order of feeding. Highprotein (all meat) diets were characterised by an increase in bacteria belonging to the Fusobacteria and Bacteroidetes phyla, whereas a high-insoluble fibre commercial diet correlated with increases in Firmicutes and Actinobacteria phyla. However, the individual dog's baseline microbiota had the most impact on the magnitude and nature of the changes in response to dietary intervention.

\section{Conclusion}

Our results suggest that the dog faecal microbiome is driven by protein and fibre composition, and targeted modification of these patterns could be useful in the modulation of the gut microbiota in different diseases.

\section{Background}

The gut microbiota is essential for maintaining health, as it exerts several beneficial effects on the host, regulates numerous biological pathways; and interacts directly and indirectly with various organs and systems in the body, including the brain, liver, bone and cardiovascular system [1]. The gut microbiota is a highly complex community that evolves rapidly and adapts to its host over a lifetime and exhibits a remarkable plasticity to environmental changes, particularly diet $[2,3]$.

Protein, carbohydrate and fat are macronutrients required in large amounts to maintain bodily functions and to provide energy for the body [2]. Diet can shape the composition of the gut microbiota as well as alter host metabolism and lipid homeostasis [4]. Even short-term dietary changes have been shown to alter human gut microbiota composition and changes can be observed within 1-3 days when there are extreme dietary modifications such as switching between an all-animal to an all-plant diet [4]. Similar studies have been performed regarding the effect of fibre on the gut microbiota of dogs [5-15]. Some studies have shown beneficial effects and changes in the gut microbiota [5, 15], whereas others have not shown any significant change $[6,12,14,16,17]$. Results have been dependent on the type of fibre, percentage of fibre, previous diet fed, duration of treatment, health status and methodology used during the analysis. Likewise, studies have also been published assessing the effect of protein [18-20], and recently with emphasis in obesity [21, 22] and raw meat diets $[8,23,24]$, but more studies are needed to understand this complex interaction under different feeding conditions.

Dysbiosis of the intestinal microbiota has been linked to chronic intestinal inflammation in people, dogs and cats [2531]. These chronic intestinal diseases are often treated by dietary modification, aimed at reducing antigenic stimulation to the intestine [32]. Hypoallergenic diets typically contain either novel protein/carbohydrate sources or hydrolysed protein sources [33]. In addition, some commercial hypoallergenic diets will have other alterations (such as increased polyunsaturated fats) compared to standard veterinary diets [34]. Hydrolysed diets are associated with beneficial changes in the intestinal microbiota and clinical signs of dogs with chronic enteropathies [35]. However, to our knowledge, there is no published data on how hypoallergenic or hydrolysed diets affect the gastrointestinal microbiome 
in healthy dogs fed different types of diets prior to the change and in turn, which component of the diet is having the most impact $[16,36,37]$. The modern pet food industry uses several fibre sources (mainly by-products derived from the processing of grains, fruits and vegetables) in the formation of diets for dogs $[15,38]$. Nevertheless, there is still a paucity of information regarding the effect of many fibre sources on the composition and activity of the intestinal microbiota of dogs and cats.

The aim of this study was to investigate the effects of dietary modification with a high-protein (all meat) diet, a highfibre diet and a hypoallergenic (hydrolysed) diet on the faecal microbiome in a cross-over trial in healthy dogs.

\section{Results}

\section{Study dogs}

Group 1 contained 10 males and 13 females with a mean age of $5.3 \pm 2.5$ (SD). Group B contained 16 males and 5 females with a mean age of $3.7 \pm 2$. Two dogs in group 1 were excluded during the feeding trial period, one due to illness, the other as it refused to eat the trial food. Four dogs were excluded from group 2 during the feeding trial period; three due to antibiotic use and one due to inadequate faecal sample collection at one time point. All dogs were fed an all meat (carcass diet) at baseline. Group 1 dogs were then fed diet sequence ACB and group 2 fed diet sequence BCA ( $A=$ hydrolysed diet; $\mathrm{B}=$ high-insoluble fibre diet; $\mathrm{C}=$ high protein, raw diet), each feeding period lasting for 6 weeks.

\section{Effect of diet on the relative abundance of bacterial groups}

From 176 samples, a total of 11,650,924 high-quality sequences were obtained, with the number of reads ranging from 12,391 to 165,430 per sample (median 60,448; mean 65,824,429; standard deviation (SD) $29,494,371$ ). Samples were rarefied at 12,390 sequences per sample for even depth of analysis.

The relative abundance of the different bacteria at phylum and family phylogenetic levels were compared among the different categories of diet. At phylum level, Firmicutes, Bacteroidetes and Fusobacteria were the most populous bacterial phyla found, as previously reported [39]. Firmicutes had a median of 44\% [range: 18-91\%] with the high-protein diet (diet C), a median of 62\% [range: $29-93 \%$ ] with the high-insoluble fibre diet (diet B) and a median of 55\% [range: 30 95\%] with the hydrolysed diet (diet A). For Bacteroidetes, the median was $14 \%$ [range: $0.22-50 \%$ ] for the high-protein diet, $16 \%$ [range: $0.44-41 \%$ ] for the high-insoluble fibre diet and $16 \%$ [range: $0.34-51 \%$ ] for the hydrolysed diet. Meanwhile, for Fusobacteria the median was $24 \%$ [range: $4-72 \%$ ] for the high-protein diet, $8 \%$ [range: $1-45 \%$ ] for the high-insoluble fibre diet and 17\% [range: 2-34\%] for the hydrolysed diet. (Figure 1A). The relative abundances at family level are shown in Figure 1B.

Next, we assessed the relative abundance of the different bacterial groups during the baseline and the washout periods (when dogs were being fed the raw meat/high protein diet) and found that the relative abundance of some phyla differed between these periods. During baseline, approximately $31 \%$ (median) of the sequences corresponded to Bacteroidetes [range: $3-50 \%$, whereas at the end of the washout period, the percentage was 5\% (median) [range: 0.22$33 \%$ ]. For Firmicutes, during baseline the percentage was 37\% [range: $18-71 \%$ ] versus $54 \%$ [range: 18-91] during the washout period (Figure 2). This difference was observed irrespective of the diet sequence for individual dogs.

Analysis of the relative abundance of the different phyla in the hydrolysed and high-insoluble fibre diets also differed between the ACB and BCA sequences. For example, samples taken from dogs at the end of the 6-week period being fed with the hydrolysed diet (diet A) showed a relative abundance of Bacteroidetes of $24 \%$ (median) [range: 0.71-51] in ACB versus $7 \%$ (median) [range: $0.34-29 \%$ ] in the CBA sequence. However, when the percentages were compared with the 
preceding diet in each diet sequence; the introduction of the hydrolysed diet did not affect the percentage of Bacteroidetes in any of the diets. In the ACB diet, the percentage of Bacteroidetes ranged between 3-50\% (median: 23\%) at baseline (high-protein, raw diet) and for the CBA diet, the percentage ranged between $0.5-33 \%$ (median: $8 \%$ ) at the end of the washout (high-protein, raw diet) period. Thus, changes should be interpreted based on the diet sequence and the preceding microbial profile of each subject, and not independently (Supplementary Figure 1, Additional file 1).

\section{Dietary effects on gut microbial alpha and beta diversity}

Alpha diversity was analysed using the Shannon index considering the subject as well as the dietary intervention (time point) and diet sequence. In general, Shannon diversity index was not affected by the change of diet when time and subject were considered; although it was lower in the washout period compared to baseline and slightly higher in BCA diet in comparison to ACB diet. The marginal $\mathrm{R}^{2}$ is about 0.3 , which suggests that the diet and sequence effects together describe about $30 \%$ of the variance in Shannon index [40] (Supplementary Table 1, Additional file 2).

In response to the diets, we see a large shift in the overall taxonomic composition of the microbiome. Beta diversity PCoA plots constructed using a weighted and an unweighted UniFrac distance matrix showed a clear separation between high-protein with the hydrolysed diet and high-insoluble fibre diets (Figure 3). The hydrolysed diet and highinsoluble fibre diets used in our study have some similar nutritional characteristics (e.g. total protein, total carbohydrate) compared to the high-protein diet that could explain, in part, the clustering pattern. PERMANOVA (Adonis) analysis showed that the type of diet explained $\sim 20 \%$ of the variability in beta- diversity $\left(R^{2}: 19, p: 0.001\right)$, whereas diet sequence only explained $1 \%$ of the variability $\left(R^{2}: 1, p: 0.007\right)$ (Supplementary Figure 2 , Additional file 3 ). When the interaction of these two factors were assessed, diet sequence explained $\sim 5 \%$ of the variability caused by the type of diet $\left(R^{2}: 6, p\right.$ : 0.001).

In accordance with the results showed in the relative abundance tables, PERMANOVA (Adonis) analysis using the Bray Curtis distance identified a significant difference in beta-diversity between the baseline and washout periods $\left(R^{2}: 25, p\right.$ : 0.001).

Analysis of each group separately, showed that the shifts of the microbiota increased over time, when compared to the baseline diet and it was independent of the diet sequence. In line, with the previous clustering pattern, the distance between the hydrolysed and high-insoluble fibre diet was lower (Supplementary Figure 3, Additional file 4: diet ACB, and supplementary figure 4, Additional file 5 diet BCA). The consistency of the community shift argues for a direct effect of the diet as, in the absence of intervention, the dog microbiota has been reported to be stable over time, using 16S rRNA profiling [41].

\section{Differential dietary effects on gut bacterial phyla and families}

A Dirichlet regression model was performed to compare the microbial differential abundance in each diet sequence considering the variation between dogs and the diets. At phylum level, the high protein diet was enriched with Fusobacteria, for the ACB diet sequence, whereas the high insoluble-fibre and hydrolysed diet induced an enrichment in the Firmicutes phylum. Firmicutes was also enriched in the washout period but not during the baseline, whereas Bacteroidetes was enriched only at baseline but not during the washout period. In addition, Actinobacteria was enriched in the high-insoluble fibre diet but only in the ACB sequence (Figure 4).

At family level, the top 20 most abundant families was assessed. Here, we could also see that the results were dependent on the diet sequence, suggesting that the outcome of a diet intervention is influenced by the previous dietary 
history and the baseline microbiome of the individual. For example, Turicibacteraceae, Lactobacillaceae, Bifidobacteriaceae and Erysipelotrichaceae were more abundant in the high-insoluble fibre group but only in the ACB diet. Peptostreptococcaceae and Clostridiaceae were more abundant in the high-protein group but only during the washout period, whereas Bacteroidaceae was more abundant in the baseline diet but not during the washout period and Fusobacteriaceae was more abundant in both periods, baseline and washout period for both sequence diets. For the hydrolysed diet, only Veillonellaceae was more abundant in comparison with the other diets, but only during the ACB diet sequence (Figure 5). Veillonellaceae has been positively correlated with fibre intake [42].

At the genus level, the ratio of Prevotella to Bacteroides has also been found to be important in the human gut microbiome; it changes in response to diet, with higher Prevotella relative abundance being observed with high carbohydrate diets, while higher relative abundance of Bacteroides has been associated with a high-protein diet [43]. In our study, we observed that the ratio of Prevotella to Bacteroides was higher in the hydrolysed and high-insoluble fibre diets compared to the high-protein diet (Supplementary figure 5, Additional file 6).

\section{Functional changes in the gut microbiota}

Phylogenetic investigation of communities by reconstruction of unobserved states (PICRUSt) was performed on the 16S rRNA gene gut bacterial composition data to predict Kyoto Encyclopedia of Genes and Genomes (KEGG) orthologs (KOs) and pathways [44]. All predicted KO pathways at L2 level were subjected to a linear mixed-effects model considering the type of diet and the diet sequence as predictors of the effect of each functional pathway. However, there were no clear effects of diet type or sequence on the predicted metagenome functional content.

\section{Discussion}

Several studies have been conducted on the effect of diet on the gut microbiota in humans and rodents, and more studies are emerging in dogs. However, the gut microbiota-diet relationship is complex and challenging to characterise as many factors may influence the outcome [2]. In our study, all dogs were receiving the same baseline diet, were the same breed, similar age, similar body condition and lived in the same environment; which served to eliminate many confounders factors that could influence the results. In general, we observed that the treatment diets had a significant residual impact on the faecal communities of all dogs and results were dependent on the composition of the gut microbiota at the start of the intervention.

Analysis of the alpha diversity between the different categories of diet, showed that the high-insoluble fibre diet and hydrolysed diet have a higher Shannon diversity in comparison with the raw meat, high-protein diet. However, when the analysis was done per subject, the difference in Shannon diversity was minimal between diets.

Studies investigating the direct impact of protein on gut microbiota composition and functionality have shown that protein quality and source are as important as total amounts in people, but less so in dogs $[2,16,44]$. Analysis of the gut microbiota showed that diet C (raw all-meat/high protein diet) in our study was characterised by an overrepresentation of bacteria belonging to the Fusobacteria phylum. This contrasts with a previous study made in obese and lean dogs with high-protein dietary intervention, where the changes in Fusobacteria were relatively small, although the levels of protein differed between studies ( $49.38 \%$ vs. $69 \%$ in ours) and was of a shorter duration [21]. Another study where the dry commercial diet was changed to minced beef, also showed minimal changes in the Fusobacteria content [18]. High levels of Fusobacterium sp. have been observed in carnivores of multiple species [3,45,46]; and in humans, increases in the levels of Fusobacterium are seen in people consuming a diet high in red and processed meats and are associated with an increased risk of development of colorectal cancer $[47,48]$. In one study of dogs fed a red meat diet for 9 weeks, an increased Fusobacterium abundance ( 15\%) was present at 6 weeks, suggesting these changes may take time to 
develop (20). Diet C in our study consisted mainly of horse carcass, which is a vastly greater percentage of protein in diet than most commercial diets (prescription or supermarket brand). Digestibility of scrap meat may be lower than for high quality protein due to the high amount of connective tissue, and digestibility of macronutrients may also influence the colonic microbiome [49]. Additionally, most commercial dog food does not contain horse protein, which may be biologically different than other sources of protein $[50,51]$.

At lower phylogenetic levels, an overrepresentation of members of the families Clostridiaceae and Peptostreptococcaceae were also found in the samples from dogs fed with the diet C. Clostridium is important for lysine and proline utilisation by the host via fermentation in the colon, while Peptostreptococci drive tryptophan and glutamate catabolism [52]. In people, an exclusively meat-based diet is frequently associated with high levels of bile-tolerant bacterial species like Bacteroides and low levels of Prevotella [43]. Of interest, Clostridiaceae and Peptostreptococcaceae were only enriched in the washout period, whereas the Bacteroidaceae family was enriched during baseline, emphasising the effects of previous diet in the microbiota profile.

Studies that have evaluated the impact of low-fibre/high-protein meat-based raw diets in the gut microbiome of healthy dogs $[23,24,53]$ have shown an overall decrease in the abundance of Firmicutes, including genera Peptostreptococcus and Faecalibacterium; and in Bacteroides and Prevotella (phylum Bacteroidetes). Conversely, other bacterial taxa were found to increase in abundance, including Proteobacteria and Fusobacteria (genus Fusobacterium) $[23,24]$, and two genera from phylum Firmicutes (Lactobacillus and Clostridium) [23, 53].

Although previous studies have identified increased levels of Enterobacteriaceae in dogs fed raw diets, we did not see enrichment of this bacterial group during this dietary intervention [53].

Another difference of the high protein diet compared to the other diets was the percentage of fat. Studies have reported that an animal-based diet high in fat (independent of protein) resulted in substantial changes to the microbiota and metabolites produced [4, 43,54]. Increases of bile-tolerant organisms [4] and members of the Proteobacteria family [55] and in the Firmicutes: Bacteroidetes ratio [56]. However, fat is not a homogenous macronutrient, and the structure and function can vary significantly among the type of fat [2]. Likewise, in an all meat diet fed in the manner in our study individual dogs may receive differing quantities of fat due to different distribution within tissue. Further studies need to be done to unravel the precise effect of fat on the gut microbiome in dogs.

Fibre has historically been classified as either soluble or insoluble, but plant cell walls often contain both, and this distinction does not always predict physiological function $[2,57]$. Although an agreement has not been reached, and several classification systems have been proposed, the US Codex Alimentarius Commission introduced the following definition of dietary fibre in 2009: 'carbohydrate (CHO) polymers with ten or more monomeric units, which are not hydrolysed by the endogenous enzymes in the small intestine of humans and belong to the following categories: (a) edible $\mathrm{CHO}$ polymers, naturally occurring in the food as consumed; (b) $\mathrm{CHO}$ polymers, obtained from food raw material by physical, enzymatic, or chemical means; and (c) synthetic $\mathrm{CHO}$ polymers [58].

The high-insoluble fibre diet (Diet B) used in this study contains 25.5\% (dry matter [DM]) insoluble fibre and 1.9\% DM soluble fibre; the total dietary fibre is therefore $27.6 \% \mathrm{DM}$ and crude fibre $16.4 \% \mathrm{DM}$. This type of diet is used for conditions such as weight loss, diabetes mellitus, chronic pancreatitis and historically for conditions liked colitis. Most standard canine diets fed for maintenance in adults contain crude fibre around 1.5-5 \% DM. Diet B in our study induced an enrichment in bacteria belonging to the Firmicutes and Actinobacteria phyla. However, at family level, Prevotellaceae (belonging to the Bacteroidetes phylum) was also enriched. This agrees with human studies, where it has been found that increased levels of Prevotella are associated with a plant-based diet rich in fibre, simple sugars, and plant-derived compounds, as they harbor genes for cellulose and xylan hydrolysis $[43,59]$. 
Obligate anaerobic bacteria (phyla Firmicutes and Bacteroidetes) encode a variety of enzymes for hydrolysing complex carbohydrates not digestible by the host, such as plant cell wall polysaccharides and resistant starch, which constitute most dietary fibres $[60,61]$. These components enter the large intestine and undergo microbial breakdown and subsequent fermentation. The major end products of microbial fermentation are short chain fatty acids (SCFAs), including butyrate, propionate, and acetate [62]. They are rapidly absorbed by the intestinal epithelial cells where they are involved in several cellular and regulatory processes with only $5 \%$ excreted in faeces $[63,64]$. Butyrate, mainly produced by Firmicutes, constitutes the main energy source for the epithelial cells [65] and plays an important role in brain function [66]. It is also known for its anti-cancer [67] and anti-inflammatory properties [63] and for its role in the development of the intestinal barrier [68].

Hypoallergenic and/or hydrolysed diets are used frequently in dogs for the treatment of putative food allergies and chronic enteropathy $[33,36,69]$. The main difference between a commercial dry diet designed for healthy dogs and a hypoallergenic diet is that the latter is composed of hydrolysed protein that decreases the probability of an immune response to protein dietary components [33]. The diet used (Diet A) is based on hydrolysed poultry, and although has lower fibre content and higher fat content than Diet B, it is similar in overall macronutrient composition to commercial maintenance diets. Evaluation of the effect of the hydrolysed diet did not show overrepresentation of any member at the phylum and family phylogenetic levels, in comparison with the other two diets. Potentially, dietary impact of hypoallergenic diets on the gut microbiota could be at functional level and not necessarily at taxonomic levels. It could lead to changes in bacterial metabolites that can promote the production of immunoregulatory metabolites, which interact with the host immune cells to promote non-responsiveness to innocuous luminal antigens (SCFAs) [62], stimulate secretory immunoglobulin $\mathrm{A}(\mathrm{s} \lg \mathrm{A})$ and $\beta$-defensins production; modulate the cytokine response or lead to an improvement of the intestinal barrier, ameliorating the clinical signs in dogs with intestinal inflammation [70]. Further studies, assessing function and strains could help us to elucidate the relevance and the role of these microbiota changes in the gut. It is interesting that these changes were different and independent from the high fibre diet, which suggests a different mechanism of action. Although we used PICRUSt to predict community functional's capabilities, we could not find an effect based on the type of diet or diet sequence.

Recent studies evaluating the effect of a hypoallergenic diet on the gut microbiome in healthy dogs and in dogs with food-responsive enteropathy showed that the impact of the diet was minimal in the microbial composition as well as in the metabolome $[16,37]$. In these studies, dogs were fed with commercial maintenance diets before the introduction of the new diet, whereas in our study the baseline diet was meat-based, which could potentially have an influence on the results. Also, the percentage of fat differed among hypoallergenic diets, with our diet being slighter higher in fat percentage.

We also saw that the magnitude and nature of the changes induced by the high fibre and hydrolysed diets varied according to the diet sequence. The initial bacterial composition, the fact that bacteria form a metabolic network and cross-feed each other and that there is significant heterogeneity within bacterial species in their ability to digest different types of fibre $[2,71]$ add complexity to the diet-microbiota interaction. In people, particularly in the case of fibre, it has been shown that an individual's baseline microbiota harbors predictive potential with regards to the effect of dietary constituents on the host [72]. Also, we should take into consideration that the proportion of one macronutrient to the total energy intake inherently influence the contribution from other macronutrients. Thus, the effect of a change in one macronutrient on the faecal microbiota is therefore a result of the combinatory effect of all the macronutrients [73]. Both, the decrease or the increase of a nutrient can contribute to the changes seen in a diet.

In our study, we observed that the ratio of Prevotella to Bacteroides was higher with the hydrolysed and high-insoluble fibre diets compared to the high-protein diet.. In accordance with this, it has been reported that a high fibre diet correlates with a microbiome consisting of polysaccharide-utilizing microbiota with lower protein fermentation products and fewer 
Bacteroides and Clostridia [74, 75]. However, when we analysed the families using the Dirichlet model, we observed that Prevotellaceae was only higher in the high-insoluble fibre diet and only in the ACB sequence, whereas members of the Bacteroidaceae family were higher in the high-protein diet but only during the baseline period.

Finally, assessment of the gut microbiota during the washout period showed that the gut microbiota of dogs did not revert to their original phylogenetic structure after six weeks. Previous studies in dogs have reported adaptation periods varying from 10 days to 4 weeks $[5,7,8,24,76]$. In our study, although the washout period was longer than previously reported, changes in the composition of the gut microbiota persisted over time. This was evidenced by sequence and diet effects and by differential results in bacterial abundance between baseline and washout periods. These changes could be permanent or there is a possibility that more time is required with the original diet to return to baseline levels. The intestinal microbiota is resistant to most environmental influences, returning rapidly to its pre-treatment state, particularly for short-term interventions [4]. Furthermore, studies have shown that it seems that long term improvements to dietary habits may be required to achieve permanent changes in the gut community structure [43]. However, this can depend on the magnitude and duration of the change [4,77]. The credible interval for the phyla and for the 20 most abundant families in each diet was quite broad. This could be due to small number size, high between-sample variability, substantial uncertainty in the taxa-specific effects, among other factors. For most families the predicted abundance was relatively low, making effects on those taxa difficult to detect.

The limitations of this study were the presence of only one breed, age (although they were evenly distributed in both groups), and potentially the manufacturing process of the commercial diets themselves could also have influenced the gut microbiota. Additionally, the alternate day feeding pattern of Day C could have influenced microbiome composition independently of the protein/digestibility $[78,79]$. Furthermore, day to day variations in microbiota occurs and in our study, faeces were only collected at a set time point [41]. Pooling samples over a collection period of several days may have been more beneficial to average out day-to-day variability but would have added more complexity to the analysis.

In addition, evaluation of microbial composition together with functional analysis (metabolomics, transcriptomics) would offer a better insight in the total effect of diet [71]. Different microbiomes have different potentials for producing certain metabolites, depending on the metabolic capabilities and metabolic interactions within the population. The fact that a bacterium harbours a gene does not imply that the gene is expressed. In the presence of different energy sources, bacteria may express genes to produce one, a group or several of these enzymes, depending on the environmental context [71]. Future studies could combine several approaches to elucidate the influence of the diet-microbiota interaction on host biology.

\section{Conclusion}

This study demonstrated that that dietary protein, fat and fibre ratios can impact the gut microbial composition. Alterations on the microbiota structure are dependent on the bacterial composition present at the time of intervention, as results were quite susceptible to study design, evidenced by sequence and diet effects. Further functional studies are required for a better understanding of the ways the dietary-microbiome crosstalk interacts with the host. This will allow in the future, the implementation of targeted and effective dietary interventions for the alleviation of microbiomeassociated diseases.

\section{Methods}

\section{Study dogs}


Fifty healthy foxhound dogs (all lean body weight, body condition score [BCS] range 3-5/9 Purina body condition score system) were enrolled in the study [80]. All dogs had up to date vaccination status and no signs of gastrointestinal disease or medication within the previous three months. All dogs enrolled underwent a full physical examination, complete blood count and biochemical profile. They were dewormed with praziquantel $200 \mathrm{mg}$, pyrantel $560 \mathrm{mg}$ and oxantel embonate $2180 \mathrm{mg}$ (Paratak ${ }^{\mathrm{TM}}$ Plus) on two separate occasions 12 weeks apart prior to commencement of the trial.

The dogs were normally fed a high protein (all meat/carcass) diet every second day. For the study, the dogs were kept in two groups of 25 each, physically separated during the study. The two groups had access to communal drinking water in their allocated yards, obtained from the same source, and were located close to each other, but had no contact with animals from the other group for the duration of the study. All environmental factors were the same for both groups (shelter, bedding, exercise area etc.).

Each group was randomly assigned to be fed one of the two experimental diets: Diet A (hypoallergenic/hydrolysed) (Hill's ${ }^{\circledR}$ Prescription Diet ${ }^{\circledR} \mathrm{z} / \mathrm{d}^{\circledR}$ Canine) or Diet B (high-insoluble fibre) (Hill's ${ }^{\mathrm{TM}}$ Prescription Diet ${ }^{\mathrm{TM}} \mathrm{w} / \mathrm{d}^{\mathrm{TM}}$ ) diet daily for six weeks. Following this, there was a washout phase of 6 weeks when dogs returned to their normal (meat carcass) diet (high protein: diet C) fed alternate days. The groups were then crossed over to receive the alternative diet for 6 weeks. Dogs were fed to maintain body weight once daily and had free access to water.

\section{Samples}

Individual faecal samples were obtained at 4 time points: baseline, after six weeks of the first diet (Diet A or B), after 6 weeks of washout (on baseline diet) and after 6 weeks on the second diet (crossing over to A or B). A total of 176 samples were collected.

\section{Diet composition and analysis}

The main source of protein in diet A was hydrolysed chicken liver, whereas for diet B the main source was soybean meal. The main source of carbohydrate $(\mathrm{CHO})$ in diet A was corn starch and cellulose and for diet $B$ was soybean meal. Regarding fibre, diet A was mainly composed of powdered cellulose and diet B of soybean meal and dried beet pulp. A detailed list of ingredients of the commercial diets can be found in Supplementary Table 2, Additional file 7.

The following analysis of diets A and B were obtained directly from the manufacturer (as diet is not same as currently produced). The content of diet C (horse meat carcass- bones, muscles, ligaments but no organs) was analysed using online diet composition and published references of horse meat composition for protein, fat, $\mathrm{CHO}$ (http://www.foodnutritiontable.com/nutritions/nutrient/?id=132. Page accessed April 2020) [50, 51].

\begin{tabular}{|c|c|c|c|c|c|c|c|c|c|c|}
\hline Diet & $\begin{array}{l}\text { Fat \% } \\
\text { Dry } \\
\text { matter } \\
\text { (DM) }\end{array}$ & $\begin{array}{l}\text { Fat } \\
\text { g/100kcal } \\
\text { ME* }^{*}\end{array}$ & $\begin{array}{l}\text { Protein } \\
\% \text { DM }\end{array}$ & $\begin{array}{l}\text { Protein } \\
\text { g/100kcal } \\
\text { ME* }^{*}\end{array}$ & $\begin{array}{l}\text { Crude } \\
\text { Fibre } \\
\% \text { DM }\end{array}$ & $\begin{array}{l}\text { Crude } \\
\text { Fibre } \\
\text { g/100kcal } \\
\text { ME* }\end{array}$ & $\begin{array}{l}\mathrm{CHO} \\
(\mathrm{NFE}) \\
\% \mathrm{DM}\end{array}$ & $\begin{array}{l}\mathrm{CHO} \\
\text { (NFE) } \\
\text { g/100kcal } \\
\mathrm{ME}^{*}\end{array}$ & $\begin{array}{l}\text { Ash } \\
\% \\
\text { DM }\end{array}$ & $\begin{array}{l}\text { Kcal/100 } \\
\text { g }\end{array}$ \\
\hline A & 14.4 & 4 & 19 & $5.3 \mathrm{~g}$ & 2.9 & 0.8 & 58.4 & 16.2 & 5.3 & 360 \\
\hline B & 8.7 & 2.91 & 19.2 & 6.4 & 16.4 & 5.5 & 50.8 & 16.99 & 5.0 & 299 \\
\hline $\mathrm{C}$ & 6.63 & 6.14 & 21.1 & 19.54 & 0 & 0 & 0 & 0 & 0.5 & 108 \\
\hline
\end{tabular}


Table 1: Nutrient composition of diet A (hydrolysed), B (high insoluble-fibre) and diet C (horse carcass, based on ref 50, 51). *As fed.

\section{Faecal DNA extraction}

All samples were collected upon voiding without contacting the environment (to avoid transfer of genetic material) or via rectal collection. Samples were refrigerated at $4^{\square} \mathrm{C}$ until transport to the laboratory, which was completed within 48 hours of sample collection. Samples were then frozen and stored at $-80^{\square} \mathrm{C}$ until processing.

Faecal DNA was extracted using the Power soil DNA isolation kit (MoBio® laboratories Catalog No. 12888-100); 250 mg of faeces were processed using the protocol for DNA isolation, detailed in the manufacturer's instructions, with some modifications. Briefly, the faecal pellet was added to a glass bead tube $(0.1 \mathrm{~mm})$ and $750 \mu \mathrm{L}$ of bead solution and $60 \mu \mathrm{L}$ of $\mathrm{C} 1$ solution were added. Then, samples were incubated at $94^{\square} \mathrm{C}$ for 10 minutes. Afterwards, tubes were placed in the PowerLyzer® 24 and were run at 3000 rpm for 45 seconds. Subsequent steps were done as indicated by the manufacturer. Extracted DNA was eluted from the spin column in $100 \mu \mathrm{L}$ of C6 solution from Mobio® $(10 \mathrm{mM}$ tris-Cl pH 8.0- 8.5). Extracted DNA was quantified and checked for purity, based on UV absorption ratios 260:280 nm and 260:230 $\mathrm{nm}$, on a ND1000 spectrometer (NanoDrop ${ }^{\mathrm{TM}}$ 2000/2000c). Samples with highly aberrant absorption ratios were reextracted.

\section{Bacterial 16S rRNA gene analysis}

Illumina sequencing of the V4 region of the bacterial 16S rRNA genes was performed using primers 515F (5'GTGCCAGCMGCCGCGGTAA-3') to 806R (5'- GGACTACVSGGGTATCTAAT-3"). Raw data was analysed using the open source software package Quantitative Insights into Microbial Ecology (QIIME) [82]. Version 1 (QIIME1, release 1.9.0). The sequence data was demultiplexed, and then quality filtered using the default settings for QIIME. Chimeras were detected and filtered from the reads using USEARCH [83] against the $97 \%$ clustered representative sequences from the Greengenes v 13.8 database [84]. The remaining sequences were clustered into Operational Taxonomic Units (OTUs) by using an open reference approach in QIIME [82].

Rarefaction plots were used to visualize adequacy of depth in the sequencing data. Measurements of Alpha (a) diversity and beta (ß)-diversity were done using QIIME1 and Phyloseq package (version1.18.1) [85]. Beta-diversity was assessed qualitatively using unweighted UniFrac; and quantitatively and Weighted UniFrac [86] and Bray-Curtis dissimilarity metrics. To calculate richness, alpha/beta diversity indexes and relative abundance; samples were rarefied at 12390 sequences per sample.

Phylogenetic investigation of communities by reconstruction of unobserved states (PICRUSt) [44] was used to predict functional gene content based on 16S rRNA gene data present in the Greengenes database and the KEGG database, using the "predict_metagenomes.py" command in PICRUSt (v1.0.0) (http://picrust.github.io/picrust/).

\section{Statistical Analyses}

Shannon index (alpha diversity) was defined as the response in a linear mixed model, which included a subject-level random intercept. Fixed effects were the diet, sequence, and interaction of diet and sequence. The model was defined using the 'Ime4' package in R [87]. Informativeness of the model was assessed using the marginal and conditional coefficients of determination as implemented in the 'muMin' package $[40,88]$. 
The same model was used for predictive functional analysis. The L2 level was chosen and the pathway was defined as the response in the linear mixed model which included a subject-level random intercept. The responses were logtransformed for the analysis. The package 'emmeans' from $\mathrm{R}$, was used for post-hoc comparisons among diets and sequences and for estimating marginal means and their $95 \%$ confidence intervals [89].

Associations between the diet, and sequence, and the relative abundance of phyla and families were assessed using a hierarchical Dirichlet regression model with the logit link function [90]. The response variables were the proportional abundances of 20 families, where Bifidobacteriaceae was the reference level, or the proportional abundances of 5 phyla, where Actinobacteria was the reference level. The sum-to-one compositional constraint in the family abundances was handled by the Dirichlet response distribution. A handful of zeros in the original abundance data, disallowed in the Dirichlet distribution, were arbitrarily adjusted, and an 'OTHER' category was generated to capture the proportion remaining (satisfying the sum-to-one constraint). Between-dog variability in the intercept for each bacterial family was estimated to accommodate the repeated-measures structure. The model was implemented in R [91] using the 'brms' package [92]. The MCMC sampling used 4 chains of 10000 iterations. Chain convergence was assessed visually and by the potential scale reduction statistic $R^{\wedge}$. Priors for the regression coefficients were set as $N(0,5)$, intended to be minimally informative. Due to interpretational difficulty associated with the interdependence of the parameter estimates across families, the final model was assessed using the posterior predicted abundances across groups and their $89 \%$ credible intervals.

\section{Abbreviations}

CHO: Carbohydrate

DM: Dry matter

LDA: Linear discriminant analysis

OTU: Operational taxonomic unit

PCoA: Principal-coordinate analysis

Picrust: Phylogenetic Investigation of Communities by Reconstruction of Unobserved States

PC1: Principal-coordinate 1

PC2: Principal-coordinate 2

SCFAs: Short chain fatty acids

SD: Standard deviation

slgA: Secretory immunoglobulin A

\section{Declarations}

\section{Ethics approval and consent to participate}

All animal procedures were done in accordance with the Animal Ethics committee of University of Melbourne. (Animal Ethics Committee approval AEC \# 1312931.1), using National Health and Medical Research Council (NHMRC) guidelines. Owner gave written consent and was able to withdraw animals from the trial at any point. 


\section{Consent for publication}

Not applicable.

\section{Availability of data and materials}

Sequence data generated during this study are available through NCBI's Sequence Read Archive under the BioProject number PRJNA641482. All other data is included in this published article and its supplementary information files.

\section{Competing interests}

The authors declare that they have no competing interests.

\section{Funding}

The authors received funding for this work from Hills Pet Nutrition and the University of Melbourne residency grant program.

\section{Authors' contributions}

Conceived and designed the experiment: CM. Performed the experiments: AP, LM. Microbial data analysis: RP, LM, AW, CM, JS. Statistical analysis: LM, AW. Drafting the paper: AP, LM, AW, CM. Paper revisions and final approval: AP, LM, RP, AW, JS, CM.

\section{Acknowledgements}

The authors acknowledge the support of Leilani Santos and Louise Baker during the experiments. We acknowledge the advice of Dr. Raphael Trouve regarding the statistical model.

\section{Open Access}

This article is distributed under the terms of the Creative Commons Attribution 4.0 International License (http://creativecommons.org/licenses/by/4.0/), which permits unrestricted use, distribution, and reproduction in any medium, provided you give appropriate credit to the original author(s) and the source, provide a link to the Creative Commons license, and indicate if changes were made. The Creative Commons Public Domain Dedication waiver (http://creativecommons.org/publicdomain/zero/1.0/) applies to the data made available in this article, unless otherwise stated.

\section{Additional Files}

The datasets supporting the conclusions of this article are included within the article and its additional files.

Supplementary Figure 1, Additional file 1: Relative abundance of bacteria before (high protein) and after the introduction of the new diet. A: Hypoallergenic (Hydrolysed protein) diet B: High-insoluble Fibre diet. Top 5 most abundant phyla. ${ }^{\text {a. }}$ 
Baseline (high protein) ${ }^{b}$ : Washout (high protein). ACB: $N: 23 n=23$ in each group. BCA: $N=21, n=21$ in each group. $N$ : number of dogs. $n$ : number of samples. Median with range.

Supplementary Table 1, Additional file 2: Estimates of the Linear mixed model for Shannon Index.

Supplementary Figure 2, Additional file 3: Bacterial beta diversity analysis of the different sequences of diet using principal-coordinate analysis ( $\mathrm{PCOA}$ ) of (A) unweighted UniFrac distance and (B) weighted UniFrac distance. The percentage of variation explained by the principal coordinates (PC1 and PC2) is indicated on the axes.

Supplementary Figure 3, Additional file 4: A: Principal coordinate analysis using Bray-Curtis dissimilarity (BC) index on diet ACB and the distributions of samples along the first principal component by diet. The percentage of variation explained by the principal coordinates (PC1 and PC2) is indicated on the axes. B: Distance boxplots of the differences in relative abundance between the baseline and the post-treatment sample from the same dog, measured as Bray-Curtis (BC) in diet ACB. Baseline and Washout correspond to the high-protein diet (HP).

Supplementary Figure 4, Additional file 5: A: Principal coordinate analysis using Bray-Curtis dissimilarity (BC) index on diet BCA and the distributions of samples along the first principal component by diet. The percentage of variation explained by the principal coordinates (PC1 and PC2) is indicated on the axes. B: Distance boxplots of the differences in relative abundance between the baseline and the post-treatment sample from the same dog, measured as Bray-Curtis (BC) in diet BCA. Baseline and Washout correspond to the high protein diet (HP).

Supplementary Figure 5, Additional file 6: A: Prevotella and B: Bacteroides relative abundances as a function of the diet. $\mathrm{C}$ : Ratios between the two genera in the different categories of diet.

Supplementary Table 2, Additional file 7: List of ingredients commercial diets

\section{References}

1. Zhang, Y.J., et al., Impacts of gut bacteria on human health and diseases. Int J Mol Sci, 2015. 16(4): p. 7493-519.

2. Mills, S., et al., Precision Nutrition and the Microbiome, Part I: Current State of the Science. Nutrients, 2019. 11(4).

3. Muegge, B.D., et al., Diet drives convergence in gut microbiome functions across mammalian phylogeny and within humans. Science, 2011. 332(6032): p. 970-4.

4. David, L.A., et al., Diet rapidly and reproducibly alters the human gut microbiome. Nature, 2014. 505(7484): p. 55963.

5. Middelbos, I.S., et al., Phylogenetic characterization of fecal microbial communities of dogs fed diets with or without supplemental dietary fiber using 454 pyrosequencing. PLoS One, 2010. 5(3): p. e9768.

6. Panasevich, M.R., et al., Modulation of the faecal microbiome of healthy adult dogs by inclusion of potato fibre in the diet. $\mathrm{Br} \mathrm{J}$ Nutr, 2015. 113(1): p. 125-33.

7. Panasevich, M.R., et al., Potato fiber as a dietary fiber source in dog foods. J Anim Sci, 2013. 91(11): p. $5344-52$.

8. Beloshapka, A.N., et al., Fecal microbial communities of healthy adult dogs fed raw meat-based diets with or without inulin or yeast cell wall extracts as assessed by 454 pyrosequencing. FEMS Microbiol Ecol, 2013. 84(3): p. 532-41.

9. Hang, I., et al., Effect of high contents of dietary animal-derived protein or carbohydrates on canine faecal microbiota. BMC Vet Res, 2012. 8: p. 90.

10. Pilla, R. and J.S. Suchodolski, The Role of the Canine Gut Microbiome and Metabolome in Health and Gastrointestinal Disease. Frontiers in veterinary science, 2020. 6: p. 498-498. 
11. Vanhoutte, T., et al., Molecular monitoring and characterization of the faecal microbiota of healthy dogs during fructan supplementation. FEMS Microbiol Lett, 2005. 249(1): p. 65-71.

12. Jia, J., et al., Investigation of the faecal microbiota associated with canine chronic diarrhoea. FEMS Microbiol Ecol, 2010. 71(2): p. 304-12.

13. Simpson, J.M., et al., Characterization of fecal bacterial populations in canines: effects of age, breed and dietary fiber. Microb Ecol, 2002. 44(2): p. 186-97.

14. Barry, K.A., et al., Low-level fructan supplementation of dogs enhances nutrient digestion and modifies stool metabolite concentrations, but does not alter fecal microbiota populations. J Anim Sci, 2009. 87(10): p. $3244-52$.

15. Biagi, G., et al., Influence of some potential prebiotics and fibre-rich foodstuffs on composition and activity of canine intestinal microbiota. Animal Feed Science and Technology, 2010. 159(1): p. 50-58.

16. Bresciani, F., et al., Effect of an extruded animal protein-free diet on fecal microbiota of dogs with food-responsive enteropathy. Journal of Veterinary Internal Medicine, 2018. 32(6): p. 1903-1910.

17. Pilla, R., et al., Administration of a Synbiotic Containing Enterococcus faecium Does Not Significantly Alter Fecal Microbiota Richness or Diversity in Dogs With and Without Food-Responsive Chronic Enteropathy. Frontiers in Veterinary Science, 2019. 6(277).

18. Herstad, K.M.V., et al., A diet change from dry food to beef induces reversible changes on the faecal microbiota in healthy, adult client-owned dogs. BMC Vet Res, 2017. 13(1): p. 147.

19. Hang, l., et al., Impact of diets with a high content of greaves-meal protein or carbohydrates on faecal characteristics, volatile fatty acids and faecal calprotectin concentrations in healthy dogs. BMC Vet Res, 2013. 9: p. 201.

20. Bermingham, E.N., et al., Key bacterial families (Clostridiaceae, Erysipelotrichaceae and Bacteroidaceae) are related to the digestion of protein and energy in dogs. PeerJ, 2017. 5: p. e3019.

21. Coelho, L.P., et al., Similarity of the dog and human gut microbiomes in gene content and response to diet. Microbiome, 2018. 6(1): p. 72.

22. Li, Q., et al., Effects of the Dietary Protein and Carbohydrate Ratio on Gut Microbiomes in Dogs of Different Body Conditions. mBio, 2017. 8(1).

23. Kim, J., et al., Differences in the gut microbiota of dogs (Canis lupus familiaris) fed a natural diet or a commercial feed revealed by the Illumina MiSeq platform. Gut Pathog, 2017. 9: p. 68.

24. Sandri, M., et al., Raw meat based diet influences faecal microbiome and end products of fermentation in healthy dogs. BMC Vet Res, 2017. 13(1): p. 65.

25. Manichanh, C., et al., The gut microbiota in IBD. Nat Rev Gastroenterol Hepatol, 2012. 9(10): p. 599-608.

26. Tilg, H., et al., The Intestinal Microbiota in Colorectal Cancer. Cancer Cell, 2018. 33(6): p. 954-964.

27. Hansen, T.H., et al., The gut microbiome in cardio-metabolic health. Genome Med, 2015. 7(1): p. 33.

28. Minamoto, Y., et al., Alteration of the fecal microbiota and serum metabolite profiles in dogs with idiopathic inflammatory bowel disease. Gut microbes, 2015. 6(1): p. 33-47.

29. AlShawaqfeh, M.K., et al., A dysbiosis index to assess microbial changes in fecal samples of dogs with chronic inflammatory enteropathy. FEMS Microbiol Ecol, 2017. 93(11).

30. Suchodolski, J.S., et al., The fecal microbiome in dogs with acute diarrhea and idiopathic inflammatory bowel disease. PLoS One, 2012. 7(12): p. e51907.

31. Honneffer, J.B., Y. Minamoto, and J.S. Suchodolski, Microbiota alterations in acute and chronic gastrointestinal inflammation of cats and dogs. World J Gastroenterol, 2014. 20(44): p. 16489-97. 
32. Simpson, K.W. and A.E. Jergens, Pitfalls and progress in the diagnosis and management of canine inflammatory bowel disease. Vet Clin North Am Small Anim Pract, 2011. 41(2): p. 381-98.

33. Cave, N.J., Hydrolyzed protein diets for dogs and cats. Vet Clin North Am Small Anim Pract, 2006. 36(6): p. 1251-68, vi.

34. Simpson, J.W., Diet and large intestinal disease in dogs and cats. J Nutr, 1998. 128(12 Suppl): p. 2717s-2722s.

35. Wang, S., et al., Diet-induced remission in chronic enteropathy is associated with altered microbial community structure and synthesis of secondary bile acids. Microbiome, 2019. 7(1): p. 126.

36. Mandigers, P.J., et al., A randomized, open-label, positively-controlled field trial of a hydrolyzed protein diet in dogs with chronic small bowel enteropathy. J Vet Intern Med, 2010. 24(6): p. 1350-7.

37. Pilla, R., et al., Effects of metronidazole on the fecal microbiome and metabolome in healthy dogs. The Journal of Veterinary Internal Medicine, 2020(Accepted for publication).

38. Graham, P.A., et al., Influence of a high fibre diet on glycaemic control and quality of life in dogs with diabetes mellitus. J Small Anim Pract, 2002. 43(2): p. 67-73.

39. Suchodolski, J.S., Intestinal microbiota of dogs and cats: a bigger world than we thought. Vet Clin North Am Small Anim Pract, 2011. 41(2): p. 261-72.

40. Nakagawa, S., P.C.D. Johnson, and H. Schielzeth, The coefficient of determination R(2) and intra-class correlation coefficient from generalized linear mixed-effects models revisited and expanded. J R Soc Interface, 2017. 14(134).

41. Garcia-Mazcorro, J.F., et al., Abundance and short-term temporal variability of fecal microbiota in healthy dogs. Microbiologyopen, 2012. 1(3): p. 340-7.

42. Hooda, S., et al., 454 pyrosequencing reveals a shift in fecal microbiota of healthy adult men consuming polydextrose or soluble corn fiber. J Nutr, 2012. 142(7): p. 1259-65.

43. Wu, G.D., et al., Linking long-term dietary patterns with gut microbial enterotypes. Science, 2011. 334(6052): p. 1058.

44. Langille, M.G.I., et al., Predictive functional profiling of microbial communities using 16S rRNA marker gene sequences. Nature Biotechnology, 2013. 31(9): p. 814-821.

45. Suchodolski, J.S., J. Camacho, and J.M. Steiner, Analysis of bacterial diversity in the canine duodenum, jejunum, ileum, and colon by comparative 16S rRNA gene analysis. FEMS Microbiol Ecol, 2008. 66(3): p. 567-78.

46. Ley, R.E., et al., Evolution of mammals and their gut microbes. Science, 2008. 320(5883): p. 1647-51.

47. Alexander, D.D. and C.A. Cushing, Red meat and colorectal cancer: a critical summary of prospective epidemiologic studies. Obes Rev, 2011. 12(5): p. e472-93.

48. Castellarin, M., et al., Fusobacterium nucleatum infection is prevalent in human colorectal carcinoma. Genome Res, 2012. 22(2): p. 299-306.

49. Brown, R.G., Digestibility of pet foods. The Canadian veterinary journal = La revue veterinaire canadienne, 1987. 28(6): p. 314-315.

50. Badiani, A., et al., Nutrient Profile of Horsemeat1. Journal of Food Composition and Analysis, 1997. 10(3): p. 254269.

51. Lorenzo, J.M., et al., Carcass characteristics, meat quality and nutritional value of horsemeat: A review. Meat Science, 2014. 96(4): p. 1478-1488.

52. Diether, N.E. and B.P. Willing, Microbial Fermentation of Dietary Protein: An Important Factor in Diet $\varangle$ Microbe『Host Interaction. Microorganisms, 2019. 7(1): p. 19.

53. Schmidt, M., et al., The fecal microbiome and metabolome differs between dogs fed Bones and Raw Food (BARF) diets and dogs fed commercial diets. PLoS One, 2018. 13(8): p. e0201279.

Page 15/21 
54. Agans, R., et al., Dietary Fatty Acids Sustain the Growth of the Human Gut Microbiota. Appl Environ Microbiol, 2018. 84(21).

55. Vaughn, A.C., et al., Energy-dense diet triggers changes in gut microbiota, reorganization of gut-brain vagal communication and increases body fat accumulation. Acta Neurobiol Exp (Wars), 2017. 77(1): p. 18-30.

56. Murphy, E.F., et al., Composition and energy harvesting capacity of the gut microbiota: relationship to diet, obesity and time in mouse models. Gut, 2010. 59(12): p. 1635-42.

57. Zmora, N., J. Suez, and E. Elinav, You are what you eat: diet, health and the gut microbiota. Nat Rev Gastroenterol Hepatol, 2019. 16(1): p. 35-56.

58. Lupton, J.R., V.A. Betteridge, and L.T. Pijls, Codex final definition of dietary fibre: Issues of implementation. Quality Assurance and Safety of Crops \& Foods, 2009. 1(4): p. 206-212.

59. De Filippo, C., et al., Impact of diet in shaping gut microbiota revealed by a comparative study in children from Europe and rural Africa. Proc Natl Acad Sci U S A, 2010. 107(33): p. 14691-6.

60. Sims, I.M., J.L. Ryan, and S.H. Kim, In vitro fermentation of prebiotic oligosaccharides by Bifidobacterium lactis HN019 and Lactobacillus spp. Anaerobe, 2014. 25: p. 11-7.

61. Hills, R.D., Jr., et al., Gut Microbiome: Profound Implications for Diet and Disease. Nutrients, 2019. 11(7): p. 1613.

62. Kasubuchi, M., et al., Dietary gut microbial metabolites, short-chain fatty acids, and host metabolic regulation. Nutrients, 2015. 7(4): p. 2839-49.

63. Lin, L. and J. Zhang, Role of intestinal microbiota and metabolites on gut homeostasis and human diseases. BMC Immunol, 2017. 18(1): p. 2.

64. Morrison, D.J. and T. Preston, Formation of short chain fatty acids by the gut microbiota and their impact on human metabolism. Gut Microbes, 2016. 7(3): p. 189-200.

65. Roediger, W.E., Role of anaerobic bacteria in the metabolic welfare of the colonic mucosa in man. Gut, 1980. 21(9): p. 793-8.

66. Stilling, R.M., et al., The neuropharmacology of butyrate: The bread and butter of the microbiota-gut-brain axis? Neurochem Int, 2016. 99: p. 110-132.

67. Wei, W., et al., Butyrate production from high-fiber diet protects against lymphoma tumor. Leuk Lymphoma, 2016. 57(10): p. 2401-8.

68. Wang, H.B., et al., Butyrate enhances intestinal epithelial barrier function via up-regulation of tight junction protein Claudin-1 transcription. Dig Dis Sci, 2012. 57(12): p. 3126-35.

69. Marks, S.L., D.P. Laflamme, and D. McAloose, Dietary trial using a commercial hypoallergenic diet containing hydrolyzed protein for dogs with inflammatory bowel disease. Vet Ther, 2002. 3(2): p. 109-18.

70. Berni Canani, R., et al., Gut Microbiome as Target for Innovative Strategies Against Food Allergy. Frontiers in immunology, 2019. 10: p. 191-191.

71. Kolodziejczyk, A.A., D. Zheng, and E. Elinav, Diet-microbiota interactions and personalized nutrition. Nat Rev Microbiol, 2019.

72. Mills, S., et al., Precision Nutrition and the Microbiome Part II: Potential Opportunities and Pathways to Commercialisation. Nutrients, 2019. 11(7).

73. Hewson-Hughes, A.K., et al., Geometric analysis of macronutrient selection in breeds of the domestic dog, Canis lupus familiaris. Behav Ecol, 2013. 24(1): p. 293-304.

74. Frame, L.A., E. Costa, and S.A. Jackson, Current explorations of nutrition and the gut microbiome: a comprehensive evaluation of the review literature. Nutrition Reviews, 2020. 
75. Christodoulides, S., et al., Systematic review with meta-analysis: effect of fibre supplementation on chronic idiopathic constipation in adults. Aliment Pharmacol Ther, 2016. 44(2): p. 103-16.

76. Kerr, K.R., et al., Effects of dietary cooked navy bean on the fecal microbiome of healthy companion dogs. PLoS One, 2013. 8(9): p. e74998.

77. Turnbaugh, P.J., et al., The effect of diet on the human gut microbiome: a metagenomic analysis in humanized gnotobiotic mice. Sci Transl Med, 2009. 1(6): p. 6ra14.

78. Kasiraj, A.C., et al., The effects of feeding and withholding food on the canine small intestinal microbiota. FEMS Microbiol Ecol, 2016. 92(6): p. fiw085.

79. Kohl, K.D., et al., Unique and shared responses of the gut microbiota to prolonged fasting: a comparative study across five classes of vertebrate hosts. FEMS Microbiology Ecology, 2014. 90(3): p. 883-894.

80. Laflamme, D., Development and validation of a body condition score system for dogs.: a clinical tool. Canine Pract, 1997. 22: p. 10-15.

81. Whitehouse-Tedd, K.M., S.L. Lefebvre, and G.P. Janssens, Dietary factors associated with faecal consistency and other indicators of gastrointestinal health in the captive cheetah (Acinonyx jubatus). PLoS One, 2015. 10(4): p. e0120903.

82. Caporaso, J.G., et al., QIIME allows analysis of high-throughput community sequencing data. Nature methods, 2010. 7(5): p. 335-336.

83. Edgar, R.C., Search and clustering orders of magnitude faster than BLAST. Bioinformatics, 2010. 26(19): p. $2460-1$.

84. DeSantis, T.Z., et al., Greengenes, a chimera-checked 16S rRNA gene database and workbench compatible with ARB. Appl Environ Microbiol, 2006. 72(7): p. 5069-72.

85. McMurdie, P.J. and S. Holmes, phyloseq: an R package for reproducible interactive analysis and graphics of microbiome census data. PLoS One, 2013. 8(4): p. e61217.

86. Lozupone, C. and R. Knight, UniFrac: a new phylogenetic method for comparing microbial communities. Appl Environ Microbiol, 2005. 71(12): p. 8228-35.

87. Bates, D., et al., Fitting Linear Mixed-Effects Models Using Ime4. Journal of Statistical Software 2015. 67(1).

88. Bartoń, K. Package 'MuMIn':Multi-Model inference. 2020; Available from: https://CRAN.Rproject.org/package=MuMIn.

89. Lenth, R., et al. Package 'emmeans': Estimated Marginal Means, aka Least-Squares Means. 2020; Available from: https://CRAN.R-project.org/package=emmeans.

90. Douma, J.C. and J.T. Weedon, Analysing continuous proportions in ecology and evolution: A practical introduction to beta and Dirichlet regression. Methods in Ecology and Evolution, 2019. 10(9): p. 1412-1430.

91. Team, R.C., R: A Language and Environment for Statistical Computing. R Foundation for Statistical Computing, Vienna. 2018.

92. Bürkner, P.-C., brms: An R Package for Bayesian Multilevel Models Using Stan. Journal of Statistical Software. 80(1).

\section{Figures}


A

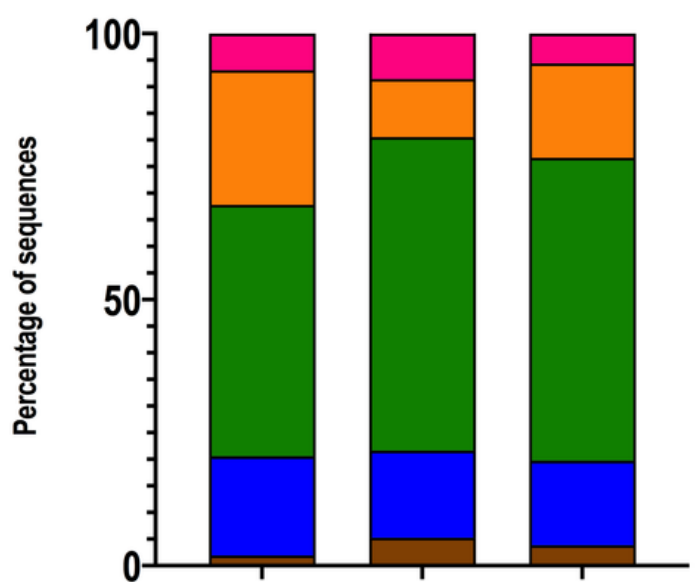

Tenericutes

Proteobacteria

Fusobacteria

$\square$ Firmicutes

$\square$ Deferribacteres

$\square$ Bacteroidetes

$\square$ Actinobacteria

$\square$ Euryarchaeota

High Protein High Fibre Hypoallergenic

B

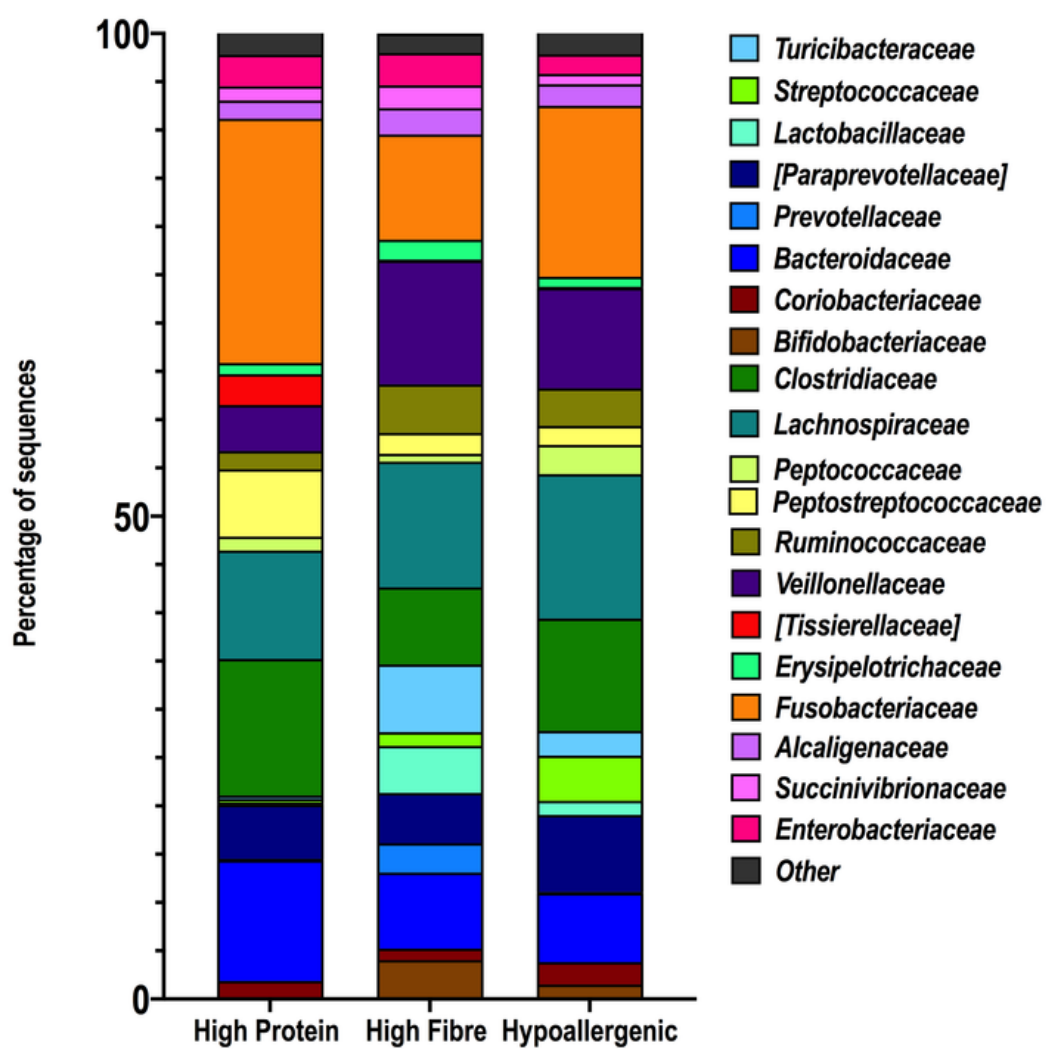

Figure 1

A: Relative abundance of bacteria at phylum level with the three diets, irrespective of sequence fed. B. Relative abundance of bacteria at family level with the three diets, irrespective of sequence fed. High-Protein: $N=44, n=88$

(baseline and washout); Hypoallergenic (hydrolysed protein) $N=44, n=44$ and High-insoluble fibre $N=44, n=44$. N: number of dogs. n: number of samples. 


\section{Baseline vs. Washout general Phylum}

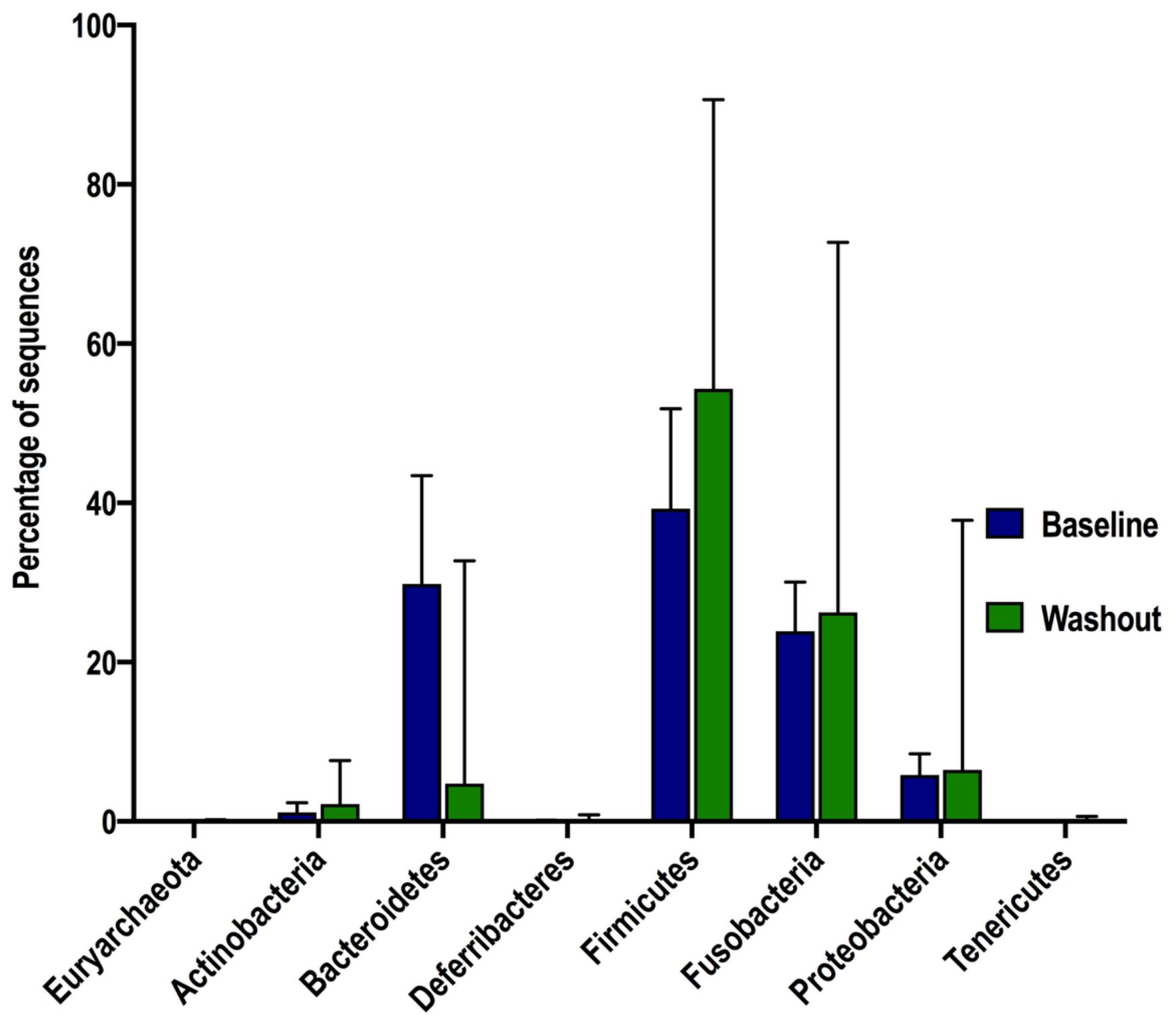

Phylum

Figure 2

Relative abundance of bacteria at phylum level Baseline versus Washout period. (High protein diet). N=44, $n=44$ each period. Median with range. N: number of dogs. n: number of samples. 

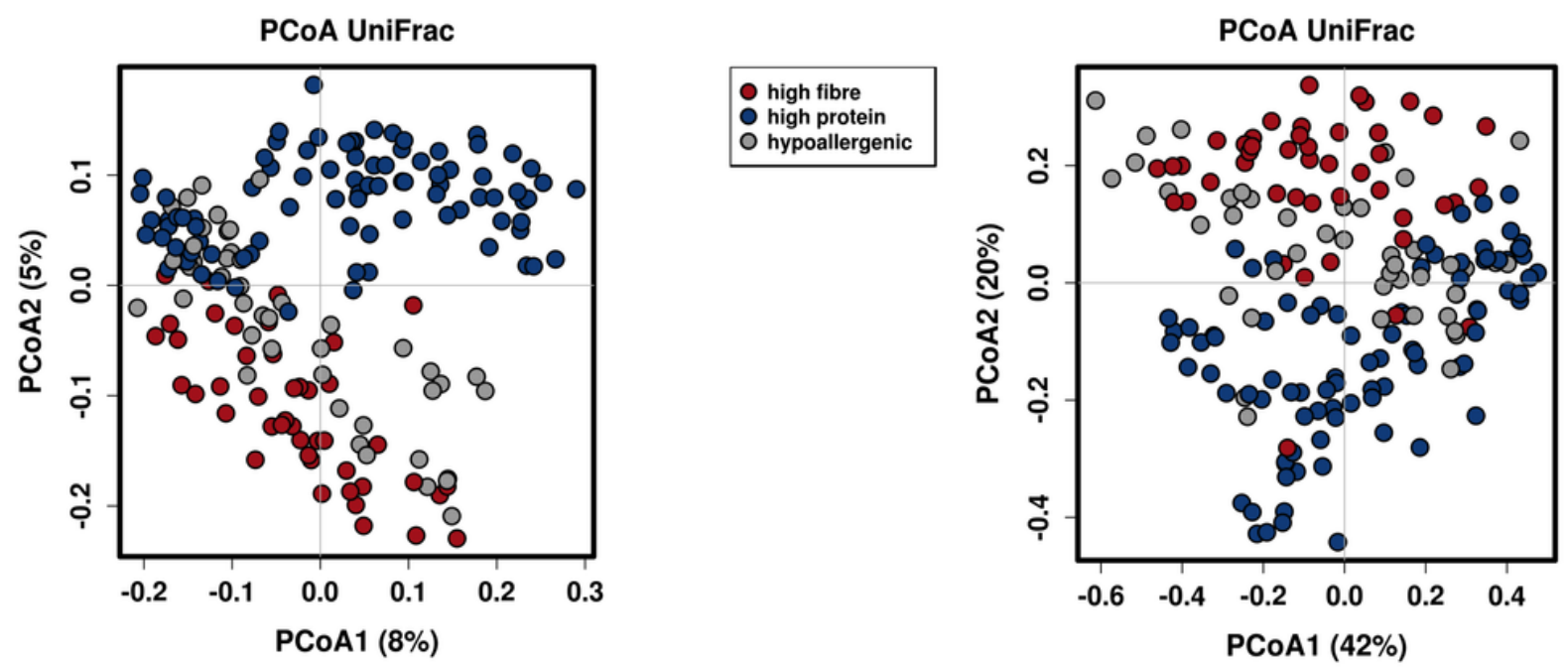

O high fibre

high protein

o hypoallergenic

Figure 3

Bacterial beta diversity analysis of the different diets using principal-coordinate analysis (PCoA) of (A) unweighted UniFrac distance and $(B)$ weighted UniFrac distance. The percentage of variation explained by the principal coordinates (PC1 and PC2) is indicated on the axes. High protein: N=44, $n=88$; hypoallergenic (hydrolysed protein) $N=44, n=44$ and high-insoluble fibre $\mathrm{N}=44, \mathrm{n}=44$. $\mathrm{N}$ : number of dogs. $\mathrm{n}$ : number of samples.

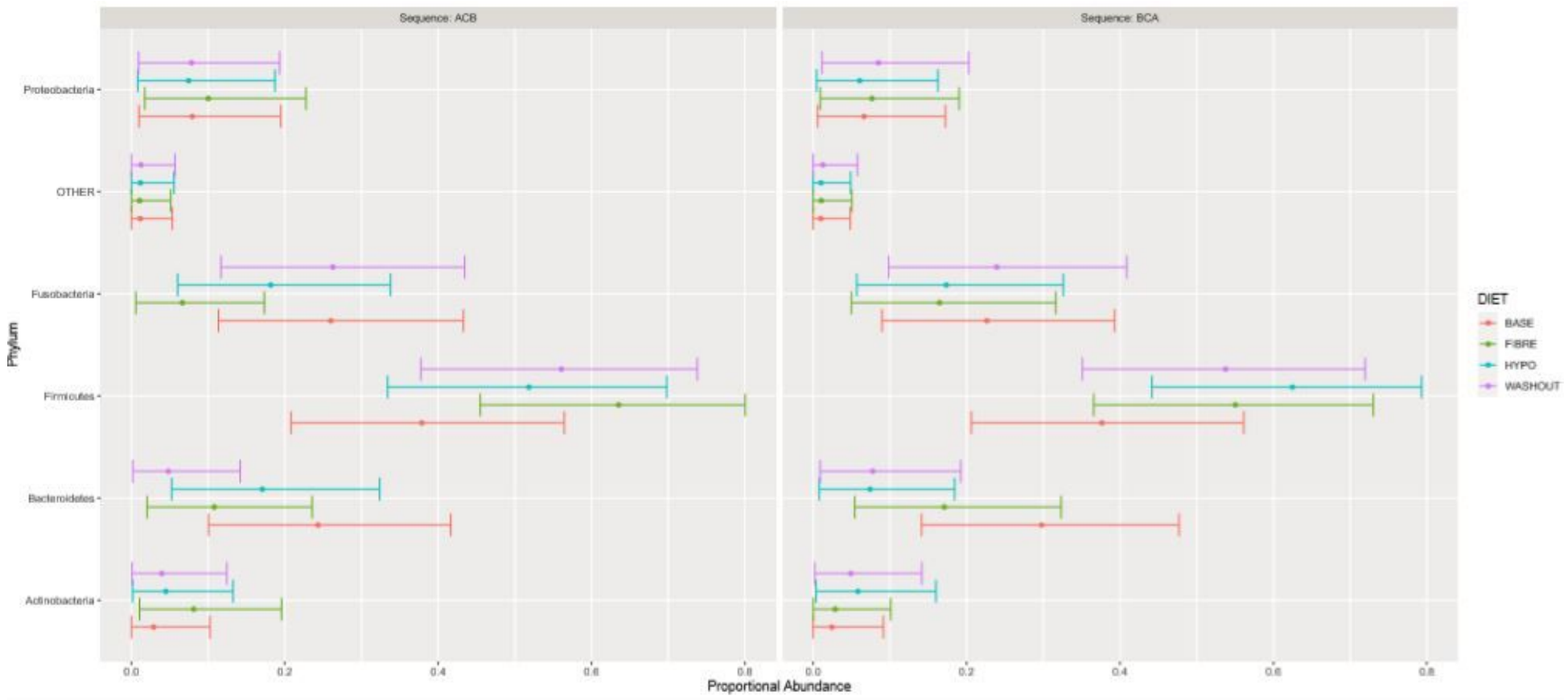

Figure 4

The posterior estimated mean relative abundances at phylum level in diet sequence $A B C$ and $B C A$. The points are the posterior mean. The bars are the $89 \%$ credible intervals. Base: Baseline (diet C), Fibre: Diet B, Hypo: Hypoallergenic (Diet A), High-insoluble Fibre (Diet B) and washout (diet C). 


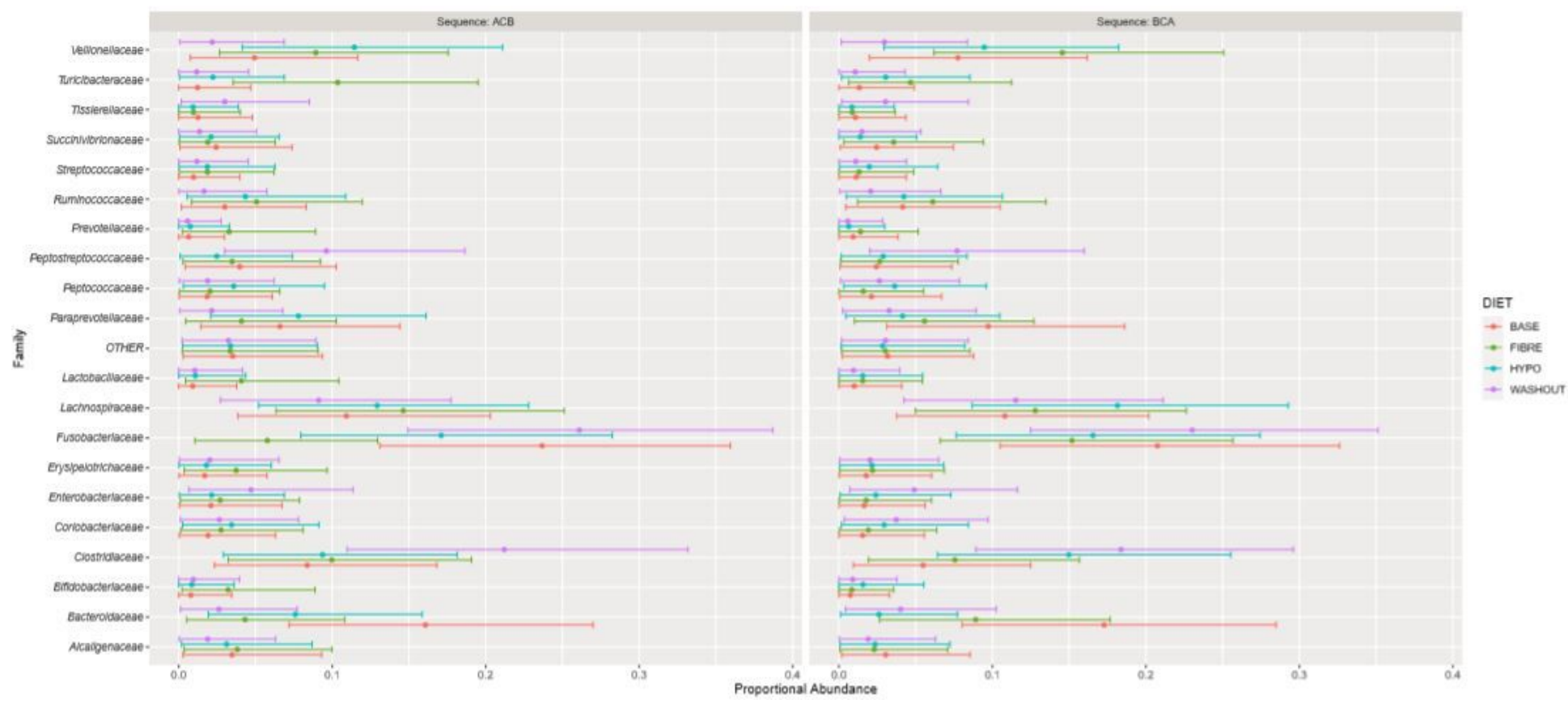

Figure 5

The posterior estimated mean relative abundances at family level in diet sequence ABC and BCA. Top of the 20 most abundant families. The points are the posterior mean. The bars are the $89 \%$ credible intervals. Base: Baseline (diet $C$ ), Fibre: Diet B, Hypo: Hypoallergenic (Diet A) High-insoluble Fibre (Diet B) and washout (diet C).

\section{Supplementary Files}

This is a list of supplementary files associated with this preprint. Click to download.

- Supplementarytable2Additionalfile7002.pdf

- SupplementaryTable1Additionalfile2.docx

- Supplementaryfigure5Additionalfile6.png

- Supplementaryfigure4Additionalfile5.png

- Supplementaryfigure3Additionalfile4.png

- SupplementaryFigure2Additionalfile3.png

- SupplementaryFigure1Additionalfile1.png 